Case Reports in
Gastroenterology
Case Rep Gastroenterol 2020;14:540-546

DOI: 10.1159/000508851

Published online: October 28, 2020

(C) 2020 The Author(s)

Published by S. Karger AG, Basel www.karger.com/crg

This article is licensed under the Creative Commons Attribution-NonCommercial 4.0 International License (CC BY-NC) (http://www.karger.com/Services/OpenAccessLicense). Usage and distribution for commercial purposes requires written permission.

\title{
Gallbladder Cancer Presenting as Mirizzi Syndrome Complicated by Rapidly Evolving 23 rRNA Gene-Linezolid Resistance with Vancomycin-Resistant Enterococcus Infection Resulting in Fatal Cholangial Sepsis
}

\author{
Daniel Schmitz ${ }^{\mathrm{a}} \quad$ Ulrich Eigner $^{\mathrm{b}} \quad$ Torsten Schmidt-Wieland $^{\mathrm{b}}$ \\ Petra Weber $^{c}$ Richard Magdeburg ${ }^{d}$ Peter Kienle ${ }^{d} \quad$ Jochen Rudi \\ aDepartment of Gastroenterology, Oncology and Diabetology, Teaching Hospital of \\ University of Heidelberg, Theresienkrankenhaus and St. Hedwig Klinik GmbH, \\ Mannheim, Germany; bepartment of Microbiology and Infectiology, Medical Service \\ Centre Laboratory Dr. Limbach and Colleagues, Heidelberg, Germany; 'Institute for \\ Hospital Hygiene, Teaching Hospital of University of Heidelberg, Theresienkrankenhaus \\ and St. Hedwig Klinik GmbH, Mannheim, Germany; Department of General and Visceral \\ Surgery, Teaching Hospital of University of Heidelberg, Theresienkrankenhaus and \\ St. Hedwig Klinik GmbH, Mannheim, Germany
}

\section{Keywords}

Mirizzi syndrome · Gallbladder cancer · Linezolid- and vancomycin-resistant enterococci ·

Case report

\section{Abstract}

We describe the case of a 71-year-old woman who presented with obstructive jaundice and subhilar bile duct stenosis. MRI showed extensive cholecystolithiasis with an impacted bile stone in the cystic duct suggesting Mirizzi syndrome. Delayed enhancement of the thickened gallbladder wall suggested inflammation instead of carcinoma. After drainage of the 


\section{Case Reports in Gastroenterology}

Case Rep Gastroenterol 2020;14:540-546 DOI: 10.1159/000508851

(c) 2020 The Author(s). Published by S. Karger AG, Basel www.karger.com/crg

Schmitz et al.: Mirizzi Syndrome Complicated by Linezolid- and Vancomycin-Resistant Enterococcus Infection

obstructed bile duct via ERCP, the patient developed liver abscesses with a nosocomial vancomycin-resistant enterococcus infection treated by linezolid. After 4 weeks, the VRE infection was complicated by a new-onset 23 rRNA gene-mediated linezolid resistance in the same bacterial strain, which was proven via core genome multilocus sequencing. Meropenem and tigecycline were administered according to a resistogram. Furthermore, percutaneous transhepatic biliary drainage of both sides of the liver was necessary. After demission, the patient had to be admitted again due to septic shock. An emergency operation revealed extended, inoperable gallbladder cancer. The patient died a few days later in the intensive care unit. An earlier diagnosis of bile duct infiltrating gallbladder cancer by cholangioscopy or laparoscopy and treatment of vancomycin-resistant enterococcus infection with daptomycin may have changed the clinical course of the disease.

\section{Background}

Gallbladder cancer or hilar cholangiocarcinoma can mimic Mirizzi syndrome and vice versa [1]. Obstructive jaundice is the leading symptom in both diseases. Frequently, the preoperative diagnosis is not correct. Endoscopic plastic stent insertion is recommended if it is presumed that the proximal common bile duct is compressed by a gallbladder or cystic duct stone. However, cholangitis may be observed as an adverse event after endoscopic retrograde cholangiopancreatography (ERCP) [2] and may delay diagnostic laparotomy or biliary surgery. Enterococci represent one of the three bacteria found most often in the bile fluid of patients with an obstructed bile duct [3]. Antimicrobial treatment of enterococci may be challenging because multidrug-resistant enterococci have been increasingly observed worldwide [4]. We report a case of gallbladder cancer presenting as Mirizzi syndrome complicated by rapidly evolving linezolid resistance in vancomycin-resistant enterococcus infection resulting in fatal cholangial sepsis.

\section{Case Presentation}

A 71-year-old female obese patient (107 kg) with type 2 diabetes was admitted to this tertiary referral hospital with obstructive jaundice. The total bilirubin level was $13.6 \mathrm{mg} / \mathrm{dL}$. Abdominal ultrasound showed multiple gallbladder stones and a large, impacted stone $(12$ $\mathrm{mm}$ ) in the cystic duct with a thickened gallbladder wall up to $17 \mathrm{~mm}$ in diameter. Magnetic resonance cholangiopancreatography demonstrated a delayed enhancement of the thickened gallbladder wall. Therefore, the thickened wall was interpreted as inflammation associated with Mirizzi syndrome (Fig. 1). An ERCP with biliary stent insertion was performed to resolve the bile duct obstruction. Cholangiography showed subhilar stenosis with consecutive intrahepatic cholestasis (Fig. 2). After 3 days, a further ERCP had to be performed due to an early stent occlusion with cholangitis (no microorganism detected). After concomitant antibiotic treatment with ceftriaxone and metronidazole, the patient was discharged with a total bilirubin level of $1.9 \mathrm{mg} / \mathrm{dL}$ during follow-up, and a readmission cholecystectomy was scheduled. Nine days later, the patient had to be readmitted due to fever and signs of cholangial sepsis with hypotension, fever, and renal failure. The CRP value was $314 \mathrm{mg} / \mathrm{dL}$. Computed tomography showed a liver abscess in seg. VII that had to be drained percutaneously (Fig. 3). Blood culture revealed an infection with Enterococcus faecalis and Streptococcus anginosus. The liver abscess culture showed a co-infection with vancomycin-resistant Enterococcus faecium (VRE), 


\section{Case Reports in Gastroenterology}

Case Rep Gastroenterol 2020;14:540-546

DOI: 10.1159/000508851

(c) 2020 The Author(s). Published by S. Karger AG, Basel www.karger.com/crg

Schmitz et al.: Mirizzi Syndrome Complicated by Linezolid- and Vancomycin-Resistant Enterococcus Infection

which was treated with meropenem and linezolid in accordance with the resistogram. Additionally, the inserted double pigtail plastic stent was exchanged with a shorter plastic stent, as it was presumed that the inserted long plastic stent had perforated a small intrahepatic bile duct that had resulted in an intrahepatic biloma abscess. Biloma was proven by an elevated total bilirubin level of $44 \mathrm{mg} / \mathrm{dL}$ in the drained abscess fluid. Upon further follow-up, the patient developed prolonged systemic VRE sepsis with pleural and articular effusions and central venous catheter infection. Four weeks after re-admission, a linezolid-vancomycin-resistant E. faecium (LVRE) was detected in the renewed liver abscess drainage. The genetic relationship of the E. faecium strains was analyzed using the core genome multilocus sequence typing method [5]. All VRE and LVRE strains that were examined belonged to the same clone, showing no allelic difference within the genes compared. The linezolid mutation of the resistant VRE strain was mediated by the G2576T mutation in the V domain of the 23 rRNA gene, which was proven by aligning the raw read sequences to the LRE finder database [6]. The infectious disease-related ward round recommended antimicrobial treatment with meropenem and tigecycline. In the meantime, further exchange of the biliary plastic stents had failed, and a double-sided percutaneous biliary drainage was successfully performed (Fig. 4). Subsequently, the patient was discharged and experienced improvements in the infection after a 2-month hospital stay. Five days later, the patient had to be re-admitted again due to cholangial septic shock. An emergency cholecystectomy with bile duct revision and t-tube insertion was performed. Histology revealed a moderate differentiated adenocarcinoma of the gallbladder at the pT3 pNX L1 V0 R1 G2 stage. Despite the emergency operation and a comprehensive intensive care unit treatment, the patient died 3 days later.

\section{Discussion and Conclusion}

It is commonly known that gallbladder cancer or subhilar cholangiocarcinoma may present as Mirizzi syndrome in the presence of an impacted cystic duct stone close to the obstructed proximal extrahepatic bile duct [1]. However, in this case, inflammation was presumed by MRI, as late contrast medium enhancement of the thickened gallbladder wall was detected. Usually, gallbladder cancer is associated with early and persistent enhancement [7]. Assuming Mirizzi syndrome, endoscopic drainage of the common bile duct was performed before cholecystectomy. Cholangioscopy with biopsy [8] or diagnostic laparoscopy or laparotomy might support the differential diagnosis between malignant and benign proximal extrahepatic bile duct stenosis. However, none of these investigations were performed in this case. However, exclusion of malignancy should be mandatory if a patient with presumed Mirizzi syndrome receives only nonsurgical treatment. In this case, cholangioscopy or early laparoscopy should have been implemented in the diagnostic routine algorithm.

However, it remains questionable whether the patient's course would have been changed in this case. Following ERCP, the patient developed cholangitis with an intrahepatic biloma abscess despite normalized cholestasis values. According to a meta-analysis, cholangitis occurs as an adverse event in 1.4\% of all ERCPs [2]. Usually, cholangitis can be easily cured with antibiotic standard therapy covering the most often documented microorganisms, such as Escherichia coli. Klebsiella and Enterococcus in accordance with common guidelines [9]. However, in this patient, the abscess contained vancomycin-resistant E. faecium. Because there was no reason for VRE screening upon hospital admission, the VRE infection had to be classified as a hospital-acquired infection. The patient received broad antimicrobial treatment that included linezolid in accordance with the resistogram. After 4 weeks, a linezolid-resistant VRE 


\section{Case Reports in Gastroenterology}

Case Rep Gastroenterol 2020;14:540-546 DOI: 10.1159/000508851

(c) 2020 The Author(s). Published by S. Karger AG, Basel www.karger.com/crg

Schmitz et al.: Mirizzi Syndrome Complicated by Linezolid- and Vancomycin-Resistant Enterococcus Infection

(LVRE) was detected in the liver abscess. Core genome multilocus sequencing showed that the VRE and LVRE strains belonged to the same clone, which demonstrates rapidly evolving linezolid resistance. Linezolid mutation of the resistant VRE strain was mediated by the G2576T mutation in the $\mathrm{V}$ domain of the 23 rRNA gene, which is the most frequent mutation causing linezolid resistance in VRE [10]. This may be due to the remarkable genetic plasticity of VRE, allowing the strains to acquire genes associated with antimicrobial resistance [11]. In particular, E. faecium develops multidrug resistance (including ampicillin, vancomycin and linezolid) more often than other Enterococcus species. Thus, E. faecium can cause severe and sometimes untreatable infections in severely ill patients [12]. There is no optimal therapy for the treatment of VRE infections. Linezolid is often used in the treatment of VRE infections. However, its bacteriostatic activity as well as recently published data suggest that the bactericide antibiotic daptomycin might be the better treatment for deep-seated VRE infections. In the well-designed retrospective cohort study from Britt et al. [13] with a total of 644 patients, linezolid was associated with a higher 30-day mortality ( 42.9 vs. $33.5 \% ; p=0.014$ ) and higher microbiologic failure rates (14.6 vs. 6.4\%; $p=0.011$ ) than daptomycin. After the calculation of confounding factors, the adjusted difference in favor of daptomycin remained unchanged. This was even more remarkable because most patients were relatively underdosed (6 mg/kg) [14]. If daptomycin is used in VRE infections, it should be combined with a $\beta$-lactam antibiotic such as ampicillin or meropenem to improve survival caused by synergistic effects [11]. In conclusion, the intravenous administration of daptomycin (daily dose $10-12 \mathrm{mg} / \mathrm{kg}$ ) plus a $\beta$-lactam antibiotic instead of linezolid plus $\beta$-lactam antibiotic may have changed the clinical course of the patient in this case report. According to an international antimicrobial surveillance program, linezolid resistance accounts for $1.4 \%$ of VRE infections and shows an increasing tendency [4]. In this patient, LVRE infection was treated with tigecycline and meropenem, resulting in temporary infection control. Tigecycline is a glycylcycline antibiotic that binds to the 30 S ribosomal subunit of E. faecium, thereby inhibiting protein synthesis of the microorganism and resulting in bacteriostatic activity. Thus, the combination of tigecycline and a bactericide antibiotic is recommended. However, the use of tigecycline is limited by adverse events such as nausea and a high volume of distribution (7-17 L/kg), causing low levels of tigecycline in the serum. In a few case reports, tigecycline was successfully combined with daptomycin with a therapy duration of 8-10 weeks for refractory endocarditis [15]. In summary, the optimal combination that would yield the best outcomes in the treatment of VRE or LVRE faecium has not yet been proven by randomized prospective studies and remains a future challenge. In conclusion, cholangiocellular carcinoma should be ruled out by cholangioscopy or early diagnostic laparoscopy in patients presenting with Mirizzi syndrome. The treatment of VRE or LVRE infection of the biliary tract with linezolid plus meropenem or tigecycline plus meropenem was insufficient in this case, resulting in fatal cholangial sepsis. Adding daptomycin to a combined antibiotic therapy might have been a better treatment.

\section{Statement of Ethics}

The case report was conducted in accordance with the Declaration of Helsinki. The spouse of the deceased patient has given his written informed consent to publish the case (including publication of images). 


\section{Case Reports in Gastroenterology}

\begin{tabular}{l|l}
\hline Case Rep Gastroenterol 2020;14:540-546 \\
\hline DOI: 10.1159/000508851 & $\begin{array}{l}\text { @ 2020 The Author(s). Published by S. Karger AG, Basel } \\
\text { www.karger.com/crg }\end{array}$ \\
\hline
\end{tabular}

Schmitz et al.: Mirizzi Syndrome Complicated by Linezolid- and Vancomycin-Resistant Enterococcus Infection

\section{Disclosure Statement}

All authors have no conflicts of interest to declare.

\section{Funding Sources}

No specific funding was received for this case report.

\section{Author Contributions}

All authors approved the final version of the manuscript and contributed to this case report as follows: D.S: data acquisition, analysis and interpretation of the data as well as manuscript preparation; U.E: data acquisition and review of the manuscript: T.S.-W: analysis and interpretation of the data and review of the manuscript; P.W: interpretation of the data and review of the manuscript; R.M: data acquisition, analysis and interpretation; P.K: data acquisition, analysis and interpretation; J.R: interpretation of the data and review of the manuscript.

\section{References}

1 Clemente G, Tringali A, De Rose AM, Panettieri E, Murazio M, Nuzzo G, et al. Mirizzi Syndrome: Diagnosis and Management of a Challenging Biliary Disease. Can J Gastroenterol Hepatol. 2018 Aug;2018:6962090.

2 Andriulli A, Loperfido S, Napolitano G, Niro G, Valvano MR, Spirito F, et al. Incidence rates of post-ERCP complications: a systematic survey of prospective studies. Am J Gastroenterol. 2007 Aug;102(8):1781-8.

3 Ruan HQ, Liao GL, Peng P, Liu SQ, Wu CL, Qin JF, et al. Microbial Profiles and Risk Factors of Preexisting Biliary Infection in Patients with Therapeutic Endoscopy. Gastroenterol Res Pract. 2019 May;2019:1527328.

4 Pfaller MA, Cormican M, Flamm RK, Mendes RE, Jones RN. Temporal and Geographic Variation in Antimicrobial Susceptibility and Resistance Patterns of Enterococci: Results From the SENTRY Antimicrobial Surveillance Program, 1997-2016. Open Forum Infect Dis. 2019;6(Suppl 1):S 54-62.

5 de Been M, Pinholt M, Top J, Bletz S, Mellmann A, van Schaik W, et al. Core Genome Multilocus Sequence Typing Scheme for High- Resolution Typing of Enterococcus faecium. J Clin Microbiol. 2015 Dec;53(12):3788-97.

6 Hasman H, Clausen PT, Kaya H, Hansen F, Knudsen JD, Wang M, et al. LRE-Finder, a Web tool for detection of the 23S rRNA mutations and the optrA, $c f r, \operatorname{cfr}(\mathrm{B})$ and poxtA genes encoding linezolid resistance in enterococci from whole-genome sequences. J Antimicrob Chemother. 2019 Jun;74(6):1473-6.

7 Catalano OA, Sahani DV, Kalva SP, Cushing MS, Hahn PF, Brown JJ, et al. MR imaging of the gallbladder: a pictorial essay. Radiographics. 2008 Jan-Feb;28(1):135-55.

8 Lee YN, Moon JH, Choi HJ, Kim HK, Lee HW, Lee TH, et al. Tissue acquisition for diagnosis of biliary strictures using peroral cholangioscopy or endoscopic ultrasound-guided fine-needle aspiration. Endoscopy. 2019 Jan;51(1):50-9.

9 Gomi H, Solomkin JS, Schlossberg D, Okamoto K, Takada T, Strasberg SM, et al. Tokyo Guidelines 2018: antimicrobial therapy for acute cholangitis and cholecystitis. J Hepatobiliary Pancreat Sci. 2018 Jan;25(1): 3-16.

10 Bi R, Qin T, Fan W, Ma P, Gu B. The emerging problem of linezolid-resistant enterococci. J Glob Antimicrob Resist. 2018 Jun;13:11-9.

11 Contreras GA, Munita JM, Arias CA. Novel Strategies for the Management of Vancomycin-Resistant Enterococcal Infections. Curr Infect Dis Rep. 2019 May;21(7):22.

12 Papanicolaou GA, Ustun C, Young JH, Chen M, Kim S, Woo Ahn K, et al. Bloodstream infection (BSI) due to Vancomycin-Resistant Enterococcus (VRE) is associated with increased mortality after hematopoietic cell transplantation for acute leukemia and myelodysplastic syndrome: a multicenter, retrospective cohort study. Clin Infect Dis. 2019;69(10):1771-9.

13 Britt NS, Potter EM, Patel N, Steed ME. Comparison of the effectiveness and safety of linezolid and daptomycin in vancomycinresistant enterococcal bloodstream infection: a National Cohort Study of Veterans Affairs Patients. Clin Infect Dis. 2015 Sep;61(6):871-8. 
Case Reports in Gastroenterology
Case Rep Gastroenterol 2020;14:540-546

DOI: 10.1159/000508851

(c) 2020 The Author(s). Published by S. Karger AG, Basel www.karger.com/crg

Schmitz et al:: Mirizzi Syndrome Complicated by Linezolid- and Vancomycin-Resistant Enterococcus Infection

14 McKinnell JA, Arias CA. Editorial Commentary: Linezolid vs Daptomycin for Vancomycin-Resistant Enterococci: The Evidence Gap Between Trials and Clinical Experience. Clin Infect Dis. 2015 Sep;61(6): 879-82.

15 Yim J, Smith JR, Rybak MJ. Role of Combination Antimicrobial Therapy for Vancomycin-Resistant Enterococcus faecium Infections: Review of the Current Evidence. Pharmacotherapy. 2017 May;37(5): $579-92$.

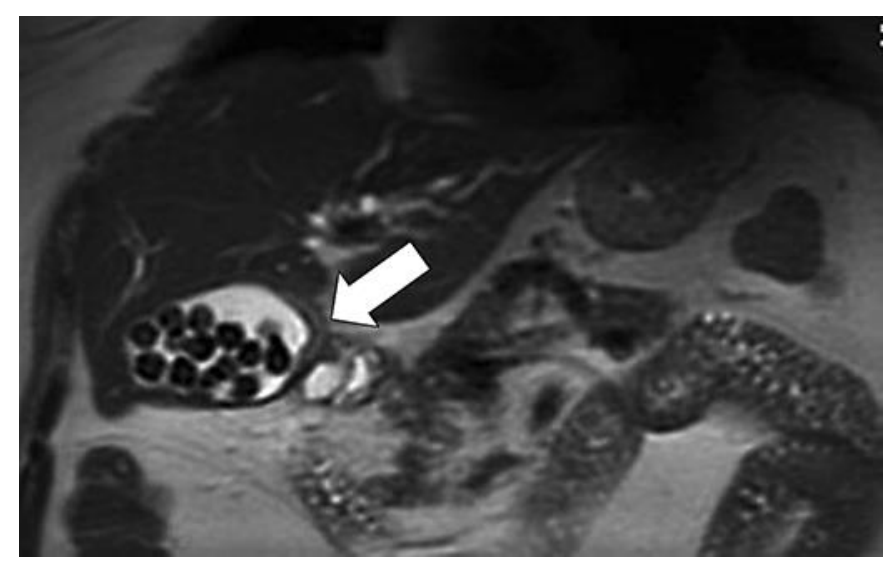

Fig. 1. MRI shows a thickened gallbladder wall with a diameter of $17 \mathrm{~mm}$ (arrow) which was interpreted as inflammation associated with the Mirizzi syndrome.

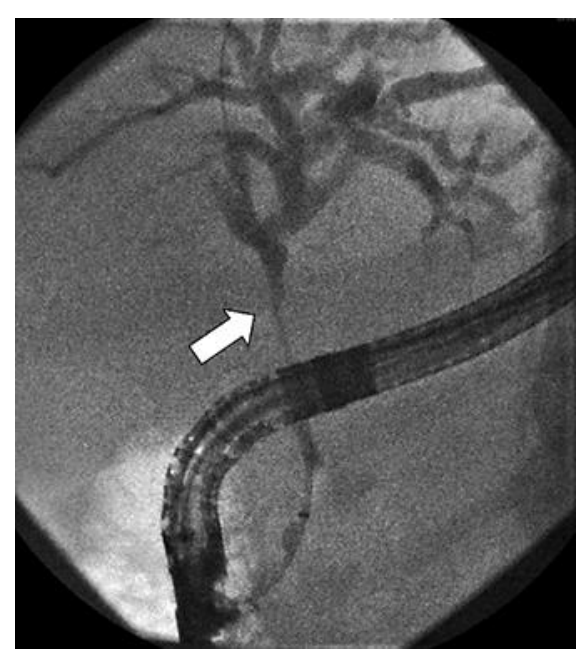

Fig. 2. ERCP cholangiography shows subhilar stenosis (arrow) of the extrahepatic common bile duct. 
Case Reports in Gastroenterology
Case Rep Gastroenterol 2020;14:540-546

DOI: $10.1159 / 000508851$

(c) 2020 The Author(s). Published by S. Karger AG, Basel www.karger.com/crg

Schmitz et al.: Mirizzi Syndrome Complicated by Linezolid- and Vancomycin-Resistant Enterococcus Infection



Fig. 3. Abdominal CT shows a liver abscess in seg. VII (arrow).

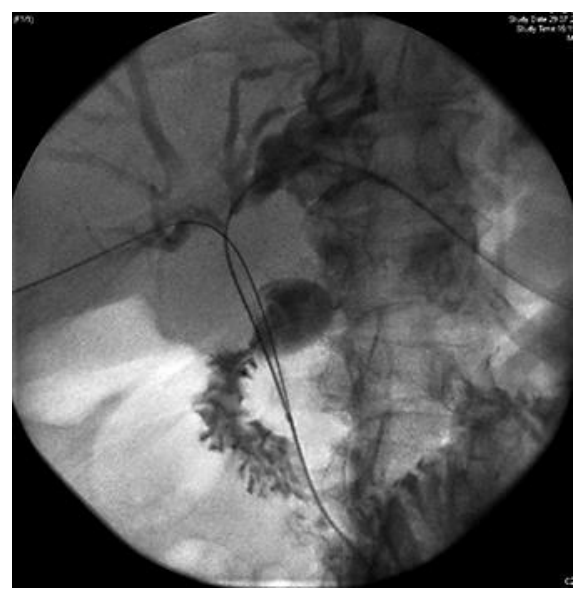

Fig. 4. Cholangiography shows double-sided percutaneous transhepatic biliary drainage in progressive hilar bile duct stenosis. 